\title{
Extracting a Purely Non-Rigid Deformation Field of a Single Structure
}

\author{
Stefanie Demirci ${ }^{1}$, Frode Manstad-Hulaas ${ }^{2}$, Nassir Navab $^{1}$ \\ ${ }^{1}$ Computer Aided Medical Procedures (CAMP), TU München, \\ ${ }^{2}$ Department of Surgery, St. Olavs Hospital, NTNU Trondheim \\ demirci@cs.tum.edu
}

\begin{abstract}
During endovascular aortic repair (EVAR) treatment, the aortic shape is subject to severe deformation that is imposed by medical instruments such as guide wires, catheters, and the stent graft. The problem definition of deformable registration of images covering the entire abdominal region, however, is highly ill-posed. We present a new method for extracting the deformation of an aneurysmatic aorta. The outline of the procedure includes initial rigid alignment of two abdominal scans, segmentation of abdominal vessel trees, and automatic reduction of their centerline structures to one specified region of interest around the aorta. Our non-rigid registration procedure then only computes local non-rigid deformation and leaves out all remaining global rigid transformations. In order to evaluate our method, experiments for the extraction of aortic deformation fields are conducted on 15 patient datasets from endovascular aortic repair (EVAR) treatment. A visual assessment of the registration results were performed by two vascular surgeons and one interventional radiologist who are all experts in EVAR procedures.
\end{abstract}

\section{Introduction}

An abdominal aortic aneurysm (AAA) is an enlargement of the abdominal aorta, resulting from weakened arterial walls. During Endovascular aortic repair (EVAR), an aortic prosthesis (stent graft) is inserted via an introducer system through one femoral artery into the aneurysmatic aorta excluding the aneurysm sack from the circulation and reducing the pressure/strain on the aortic wall.

Organ deformation in general can be measured by non-rigidly registering the preoperative to the postoperative CT scan [1]. In the case of abdominal aortic aneurysms, however, this task is associated with several problems and challenges. The overall deformation in the abdominal region includes breathing deformation and specific motion of several organs, each different and not comparable. Hence, true deformation fields of the aorta can only be obtained by registering segmentations of the pre- and postoperative structures. However, in contrasted CT scans, a segmentation of the entire vessel tree may include regions that are again subject to different types of deformations. Additionally, the implanted stent graft separates parts of the enlarged aorta from blood flow which would be computed as a shrinking deformation by the registration algorithm. As we are interested 
in the true deformation of the aorta, our method has to narrow down the vessel tree segmentation to a region of interest around the aorta and also eliminate any additional information such as shrinking and rigid body transformations due to different patient positioning.

We propose a method for extracting specific deformation of the aorta during and after EVAR. It segments both preoperative and postoperative aortic structures with minimal user interaction and automatically extracts the region of interest around the aneurysm. To this end, the centerlines of both structures are extracted and reduced to the region of interest around the aorta. After an initial rigid registration, non-linear registration of the structures provides pure deformation fields of the aorta. Our method was tested by evaluating 15 datasets of patients that were treated by EVAR.

\section{Materials and methods}

\section{$2.1 \quad$ Rigid registration}

A rigid registration problem can be formulated as the maximization of a similarity measure using image information over a possible range of transformation parameters:

$$
\mathbf{T}_{\text {opt }}=\arg \max _{\mathbf{T}} \mathcal{D}_{1}\left(\mathbf{I}^{F}, \mathbf{T} * \mathbf{I}^{M}\right)
$$

where $\mathbf{I}^{F}$ and $\mathbf{I}^{M}$ are the fixed and the moving volume respectively, $\mathbf{T}$ a rigid transformation matrix and $\mathcal{D}_{1}$ a certain similarity measure that is to be maximized. As our two CTA volumes contain severe dissimilarity introduced by the high intensities of the stent graft, an appropriate similarity measure needs to be chosen carefully. Given a segmentation of the stent graft, the adapted normalized cross correlation (A-NCC) [2] measure only evaluates those voxels that are not part of the segmentation. In our case, a rough segmentation of the stent graft can be easily extracted from the postoperative scan by thresholding. The resulting optimal transformation $\mathbf{T}_{\text {opt }}$ that maximizes A-NCC, describes the overall rigid body transformation. For our following explanations, we set $\mathbf{I}^{M}:=\mathbf{T}_{\mathrm{opt}} * \mathbf{I}^{M}$.

\subsection{Segmentation and preprocessing}

We segment the contrasted vessel tree out of the preoperative as well as the postoperative volume using the connected threshold segmentation method provided by ITK ${ }^{1}$. Given this segmentation $S$ of the abdominal vessel tree and a point bif for the aortic bifurcation, topological thinning is processed onto $S$ resulting in a centerline structure $C$ for the entire abdominal vessel tree. Then, the root seed of the aortic centerline is calculated. Here, the biggest connected component of segmented pixels in the first slice of the segmentation image is computed by counting neighboring pixels of value other than 0 . The centerline voxel nearest

\footnotetext{
1 Insight Toolkit, http://www.itk.org
} 
to the center of mass of the biggest component (assumed to be the aortic lumen) is then taken as seed point. In order to further narrow the information down to the region of interest around the aorta, the centerline structure $C$ is processed by an adapted wave front propagation algorithm. It adapts the wave propagation approach of Zahlten et al. [3] by stepping down the aortic centerline from a given seed point seed in region growing manner until the indicated bifurcation bif is reached. At all other bifurcations, our algorithm automatically finds the path belonging to the aorta by comparing the different angles between the connecting parts. The aortic path usually continues in the direction that forms the smallest angle with the previous direction. The branching paths are included into the aortic centerline data only up to the next bifurcation respectively. Thereby, we ensure a fully three-dimensional tree structure $T$ which can be used to cover a full transformation including rotation. Finally, we compute a Danielsson distance map [4] $\mathbf{D}$ on the reduced centerline data.

\subsection{Non-rigid registration}

The preprocessing steps narrow down the scatter of the abdominal vessel tree to one centerline including the aorta and small parts of all branching vessels. We can now employ a non-rigid registration in order to obtain the deformation of the aorta. Non-rigid registration consists of deforming a source image $\mathbf{I}^{M}$ to best match a target image $\mathbf{I}^{F}$. Using similar formulation as before, this can be expressed as

$$
\mathbf{V}_{\text {opt }}=\arg \min _{\mathbf{V}} \mathcal{E}\left(\mathbf{I}^{F}, \mathbf{I}^{M}, \mathbf{V}\right)
$$

There energy functional $\mathcal{E}$ is defined as

$$
\mathcal{E}\left(\mathbf{I}^{F}, \mathbf{I}^{M}, \mathbf{V}\right)=\mathcal{D}_{2}\left(\mathbf{I}^{F}, \mathbf{V}+\mathbf{I}^{M}\right)+\alpha \mathcal{S}(\mathbf{V})
$$

Here, $\mathbf{V}=\left\{\mathbf{V}_{x}, \mathbf{V}_{y}, \mathbf{V}_{z}\right\}$ gives the deformation or displacement field that warps image $\mathbf{I}^{M}$. $\mathcal{D}$ is a certain distance or dissimilarity measure and $\mathcal{S}$ a certain diffusion regularization term which ensures smooth resulting deformations, by penalizing high gradients of the displacement fields. The positive scalar $\alpha$ controls the influence of the regularization terms. In order to define the dissimilarity measure $\mathcal{D}_{2}$ for centerlines, correspondences of pre- and postoperative centerline points are required. Except of the bifurcation points, it is highly costly to establish correspondences for the other points on the centerlines. We avoid the necessity of correspondences by computing distance maps $\mathbf{D}^{F}, \mathbf{D}^{M}$ on the reduced centerline images and computing the sum of absolute differences

$$
\mathcal{D}\left(\mathbf{D}^{F}, \mathbf{V}+\mathbf{D}^{M}\right)=\sum_{x}\left|\mathbf{D}^{F}(x)-\mathbf{V}+\mathbf{D}^{M}(x)\right|
$$

as dissimilarity measure. As smoothing term for the energy functional (3), we use diffusion regularization[5].

With initial rigid registration and final non-rigid-registration, we have obtained the linear and non-linear part of the overall transformation of the abdominal aorta. However, according to Zikic et al. [6], deformation fields that 
are computed by a local nonlinear registration algorithm, still contain a linear transformation $\mathbf{T}_{\text {local }}=\mathbf{T}_{\text {linear }} \circ \mathbf{T}_{\text {nonlinear }}$. In order to obtain a pure non-linear deformation field, the authors suggest to minimize the norm of the displacement field $\mathbf{V}$ of the nonlinear component $\mathbf{T}_{\text {nonlinear }}$ with respect to the linear transformation $\mathbf{T}_{\text {linear }}$.

$$
\min _{p} \mathcal{E}\left(\mathbf{T}_{\text {linear }}\right)=\min _{p} \frac{1}{n} \sum_{i=1}^{n}\left\|\mathbf{X}_{i}-\mathbf{T}_{\text {linear }}^{-1}\left(\mathbf{Y}_{i}\right)\right\|^{2}
$$

where $\mathbf{X}$ defines a control point grid of size $n$ over $\mathbf{T}_{\text {global }}\left(\mathbf{I}^{M}\right)$ and $\mathbf{Y}=\mathbf{X}+\mathbf{V}$ presents the deformed control point grid. As we are only interested in the deformation of the aortic shape, the optimizer needs only to evaluate those displacement positions that belong to the aorta. Once the optimal linear transformation $\mathbf{T}_{\text {linear }}$ is computed, we obtain the corresponding minimal displacement field by

$$
\mathbf{V}_{\text {minimal }}(\mathbf{X})=\mathbf{T}_{\text {linear }}^{-1}(\mathbf{Y})-\mathbf{X}
$$

\section{Results}

Experiments have been conducted on 15 patient datasets each consisting of one preoperative and one postoperative CT angiography (CTA) scan. All images were acquired by a Siemens Somatom Sensation 64 scanner at our partner clinic. The reconstructed image resolution is $0.54 \times 0.54 \times 1.00 \mathrm{~mm}$.

As we deal with real medical images, there is no ground truth data available that could be used for the assessment of the registration accuracy. Therefore, we reconstruct centerlines out of the deformed distance maps and evaluate these to the preoperative and postoperative lines. Next to a visual evaluation of the registration error, we choose to use the euclidean distance between the centerlines before and after non-rigid registration.

Table 1 shows the evaluation results. For each of the 15 datasets the initial distance and final distance each presented by their maximal and mean value. Additionally, the initial and final values for the mean $\mu$, variance $\sigma^{2}$, and standard deviation $\sigma$ over all datasets are given. Our method results in reduced values (final distance) for the distance in between the respective shapes. This states that the deformed centerline fits better to the entire shape of the postoperative centerline after non-rigid registration.

The visual assessment of registration accuracy was performed by three experts from our partner medical centers (two interventional radiologists and one vascular surgeon). They were asked to evaluate four different visualizations of the non-rigid registration results and compare these to visualizations given by existing rigid registration frameworks [2]. All experts found our visualizations as highly beneficial for assessing and quantifying the aortic deformation. In contrast to the vascular surgeon to whom the branching vessels were of no importance, the interventional radiologists were also interested in the accuracy of especially the renal arteries as these are often included within the stent graft 
Table 1. Evaluation results

\begin{tabular}{|c|c|c|c|c|c|c|c|c|}
\hline & \multicolumn{2}{|c|}{ initial distance } & \multicolumn{2}{|c|}{ final distance } & \multicolumn{3}{|c|}{ bifurcation distance } & \multirow[b]{2}{*}{ bif4 } \\
\hline & $\max$ & mean & $\max$ & mean & bif1 & bif2 & bif3 & \\
\hline Dataset 1 & 11,56 & 3,46 & 5,22 & 1,14 & 6,91 & - & 6,90 & 5,45 \\
\hline Dataset 2 & 14,13 & 5,41 & 4,35 & 1,41 & 4,97 & 3,29 & 7,08 & 12,90 \\
\hline Dataset 3 & 29,68 & 5,27 & 6,77 & 1,50 & 2,89 & 3,99 & 4,39 & 11,61 \\
\hline Dataset 4 & 15,46 & 4,17 & 2,15 & 1,32 & 5,45 & 4,69 & 3,67 & 5,45 \\
\hline Dataset 5 & 16,96 & 5,41 & 4,73 & 1,14 & 10,32 & 9,92 & 7,37 & 3,38 \\
\hline Dataset 6 & 10,35 & 4,46 & 3,42 & 1,31 & 1,02 & 2,33 & 4,50 & 12,84 \\
\hline Dataset 7 & 11,21 & 4,27 & 4,11 & 1,60 & 12,45 & 11,10 & 11,13 & 5,92 \\
\hline Dataset 8 & 12,26 & 4,17 & 3,10 & 1,32 & 3,06 & 5,48 & 4,32 & 20,32 \\
\hline Dataset 9 & 17,44 & 4,46 & 3,56 & 1,24 & 15,78 & 12,95 & 11,52 & 3,05 \\
\hline Dataset 10 & 19,91 & 3,41 & 4,05 & 1,41 & 14,10 & 10,09 & 9,64 & 4,12 \\
\hline Dataset 11 & 26,00 & 5,27 & 7,41 & 1,50 & 13,77 & 10,14 & 10,58 & 2,43 \\
\hline Dataset 12 & 15,60 & 3,17 & 2,09 & 1,22 & 5,09 & 7,55 & 6,32 & 8,88 \\
\hline Dataset 13 & 9,55 & 3,46 & 4,45 & 1,14 & 3,01 & 4,99 & 4,12 & 13,83 \\
\hline Dataset 14 & 16,22 & 5,11 & 4,07 & 1,51 & 1,45 & 4,50 & 3,77 & 9,82 \\
\hline Dataset 15 & 13,08 & 5,27 & 3,10 & 1,50 & 3,78 & 6,32 & 4,51 & 10,74 \\
\hline$\mu$ & 15,96 & 4,45 & 4,17 & 1,35 & 6,93 & 6,97 & 6,65 & 8,68 \\
\hline$\sigma$ & 5,66 & 0,81 & 1,47 & 0,15 & 4,99 & 3,34 & 2,83 & 5,06 \\
\hline$\sigma^{2}$ & 32,01 & 0,66 & 2,17 & 0,02 & 24,92 & 11,17 & 8,03 & 25,61 \\
\hline
\end{tabular}

as side branches. However, as our approach highly concentrates on the aortic trunk and only takes small parts of branching vessels into account for rotational orientation purposes, branching vessels might not be accurately aligned.

\section{Discussion}

In this paper, we address the challenging task of extracting the intraoperative aortic deformation out of pre- and postoperative CT scans. We believe due to constraining the given image information to the region of interest around the aorta, it is possible to only extract aorta specific displacement fields. The method was successfully tested on 15 datasets of patient that had been treated by EVAR in the abdomen.

In the near future, we plan to optimize the non-rigid registration algorithm by establishing correspondences in order to give branching and bifurcation points more weight. Moreover, we intent to apply this method to preoperative CTA and intraoperative rotational angiography volumes in order to display electromagnetically tracked catheter positions within a combined volume. 


\section{References}

1. Crum WR, Hartkens T, Hill DLG. Non-rigid image registration: Theory and practice. Br J Radiol. 2004;77:S140-S153.

2. Demirci S, Kutter O, Manstad-Hulaas F, et al. Advanced 2D-3D registration for Endovascular Aortic Interventions: Addressing Dissimilarity in Images. Proc SPIE. 2008; p. 69182S-69182S-8.

3. Zahlten C, Juergens H, Peitgen HO. Reconstruction of branching blood vessels from ct data. In: Eurographics Workshop of Visualization in Scientific Computing. Springer; 1994. p. 161-168.

4. Danielsson PE. Euclidean Distance Mapping. Computer Graph Image Process. 1980;14(3):227-248.

5. Modersitzki J. Numerical Methods for Image Registration. Oxford University Press; 2004.

6. Zikic D, Sass Hansen M, Glocker B, et al. Computing Minimal Deformations: Application to Construction of Statistical Shape Models. Proc IEEE Conf: Computer Vision and Pattern Recognition. 2008; p. 1-8. 